\title{
Quantification of sarcomatoid differentiation in renal cell carcinoma on magnetic resonance imaging
}

\author{
Daniel Jeong ${ }^{1}$, Natarajan Raghunand ${ }^{1}$, Diego Hernando ${ }^{2}$, Michael Poch ${ }^{3}$, Katherine Jeong ${ }^{4}$, Brendan \\ Eck $^{5}$, Jasreman Dhillon ${ }^{6}$ \\ ${ }^{1}$ Department of Diagnostic Imaging and Interventional Radiology, H. Lee Moffitt Cancer Center and Research Institute, Tampa, FL, USA; \\ ${ }^{2}$ Department of Radiology, University of Wisconsin-Madison. Madison, WI, USA; ${ }^{3}$ Department of Genitourinary Oncology, H. Lee Moffitt Cancer \\ Center and Research Institute, Tampa, FL, USA; ${ }^{4}$ Department of Internal Hospital Medicine, H. Lee. Moffitt Cancer Center and Research Institute, \\ Tampa, FL, USA; ${ }^{5}$ Department of Biomedical Engineering, Case Western Reserve University, Cleveland, OH, USA; ${ }^{6}$ Department of Anatomical \\ Pathology, H. Lee Moffitt Cancer Center and Research Institute, Tampa, FL, USA
}

Correspondence to: Daniel Jeong, MD, MS. Department of Radiology, Moffitt Cancer Center, 12902 USF Magnolia Drive, Tampa, FL 33612, USA. Email: Daniel.jeong@moffitt.org.

Background: Sarcomatoid differentiation in renal cell carcinoma (sRCC) is histologically characterized by anaplastic changes of renal cell carcinoma (RCC) subtypes, which has been associated with a poor prognosis. sRCC is managed more aggressively than RCC without sarcomatoid components, so pre-operative detection of sarcomatoid differentiation would significantly affect surgical management. The purpose of this study is to compare the quantification of sarcomatoid features in RCCs on pre-operative magnetic resonance imaging (MRI) to standard histological examination.

Methods: Patients who had nephrectomy at our institution between 2000 and 2015 with pathology proven RCC and pre-operative contrast enhanced MRI abdominal scans were retrospectively reviewed. A custom MATLAB routine calculated the portion of each manually segmented whole tumor with MRI signal suggestive of sarcomatoid involvement based on prior research (MRI\%SARC). The primary endpoint compared MRI\%SARC to percent sarcomatoid involvement estimated by histological examination (HIST\%SARC) using Pearson correlation and Bland Altman analysis.

Results: A total of 17 patients with sRCC (10 males, age $60.3 \pm 11.1$ years) and 17 consecutive control patients with clear cell RCC (ccRCC) without sarcomatoid components (10 males, age 64.5 \pm 7.6 years) were evaluated. Pearson correlation analysis revealed a strong association between MRI\%SARC and HIST\%SARC $(\mathrm{r}=0.782, \mathrm{P}<0.001)$. Bland-Altman analysis demonstrated proportional bias, with a mean bias of 19.29 [95\% confidence interval (CI): 9.79-28.79] and with 95\% limits of agreement of -16.93 (95\% CI: -33.38 to -0.48 ) to 55.51 (95\% CI: 39.06-71.96), suggesting MRI\% SARC underestimated values compared to HIST\%SARC by $19 \%$.

Conclusions: Multiparametric pre-operative MRI analysis to quantify sarcomatoid features in RCC correlates with standard histological examination but underestimates percent sarcomatoid involvement.

Keywords: Magnetic resonance imaging (MRI); renal cell carcinoma (RCC); sarcomatoid

Submitted Feb 11, 2018. Accepted for publication Apr 20, 2018.

doi: 10.21037/qims.2018.04.09

View this article at: http://dx.doi.org/10.21037/qims.2018.04.09 


\section{Introduction}

Renal cell carcinoma (RCC) accounts for 90-95\% of renal tumors in adults, and about $2-3 \%$ of all adult cancers (1-3). Reported in approximately $5-10 \%$ of all RCCs, sarcomatoid differentiation in renal cell carcinoma ( $\mathrm{RCC}$ ) is histologically characterized by the anaplastic transformation of RCC subtypes (4-6). These sarcomatoid features include pleomorphic spindle-like cells, high cellularity, and cellular atypia (4). Sarcomatoid differentiation is often associated with highly aggressive behavior, rapid recurrence, and a poor prognosis (5-9). RCC patients with a greater percentage of sarcomatoid components have been found to have a higher risk of death $(9,10)$.

Currently there are no standardized pre-operative methods to determine whether a RCC has sarcomatoid features, which poses a significant dilemma of determining an appropriate clinical management strategy $(4,11,12)$. However, Schieda et al. found several features more commonly associated with sRCC, including large tumor size, the presence of peritumoral neovascularity, and larger peritumoral vessels, though any subtypes may demonstrate these characteristics (13).

Takeuchi et al. reported that sarcomatoid regions may be distinguishable from non-sarcomatoid regions of clear cell RCC (ccRCC) on T2-weighted (T2W) magnetic resonance imaging (MRI) and apparent diffusion coefficient (ADC) map appearance $(14,15)$. The authors noted that sarcomatoid components were more likely to have a T2W signal hypointense to contralateral normal renal cortex, defined as T2 low intensity areas (T2LIA), while ccRCC tumors were more likely to exhibit heterogeneous isointense to high T2 signal intensity compared to the normal renal cortex, defined as T2 high intensity areas (T2HIA) (14). In a follow-up study, Takeuchi et al. reported that while the optimal signal intensity ratio threshold for separating T2LIA from T2HIA yielded excellent sensitivity for sRCC, the specificity was poor (15).

Sarcomatoid differentiation in RCC alters clinical management once the diagnosis is known $(4,13)$. Observation is not recommended for sRCC given its aggressive nature, and other conservative strategies including ablation and partial nephrectomies with renal preservation may be effective for small or localized renal tumors but are often discouraged in patients with sRCC $(4,13)$. Radical nephrectomy in advanced non-disseminated RCC and cytoreductive nephrectomy prior to immunotherapy in metastatic RCC are the current standard of care for RCCs without sarcomatoid features $(4,13,16)$. However, in patients with known sRCC who undergo radical nephrectomy, extended lymph node dissection even in the absence of clinical disease is also recommended (17). Furthermore, in patients with metastatic RCC who are known to have sarcomatoid components, cytoreductive nephrectomy has little added survival benefit and may delay the initiation of systemic therapy $(4,18)$.

Therefore, pre-operative knowledge of sarcomatoid differentiation within a RCC tumor would significantly affect surgical management. To our knowledge, the use of MRI to localize and quantitatively measure the degree of sarcomatoid components in RCC has not been extensively reported. The purpose of this study is to apply quantitative imaging analysis techniques on pre-operative MRI in patients with sRCC to detect sarcomatoid components and compare these values to gold standard post-operative histological examination.

\section{Methods}

\section{Patient selection}

A retrospective review was performed of 34 patients with pathology proven RCC and available pre-operative contrast enhanced MRI abdomen scans for this single center cohort study. Institutional Review Board approval was obtained and waiver of consent was granted. Data was acquired in compliance with all applicable Health Insurance Portability and Accountability Act regulations, and research was performed according to the Declaration of Helsinki principles.

All patients who had surgical resection of RCC at our institution between 2000 and 2015 were reviewed. Patients who did not have pre-operative MRI scans within 3 months of a nephrectomy or where histology slides were not available for review were excluded from our study. A total of 17 subjects with histology positive for RCC with sarcomatoid differentiation also had available pre-operative MRI scans, and these comprised our sRCC cohort. In addition, 17 consecutively chosen patients with ccRCC without sarcomatoid differentiation and with available pre-nephrectomy MRI scans served as the control group (ccRCC). Medical records were reviewed for patient demographics, tumor location, grade, lymph node status, use of systemic therapy, and surgical pathology.

\section{Pathologic evaluation}

Hematoxylin and eosin-stained slides of resected tumors 
were reviewed by a dedicated genitourinary pathologist. Pathologic variables including tumor size and stage, necrosis or hemorrhage, percentage sarcomatoid change, and lymph node status were considered. At least 5-8 regions within each tumor were examined. The fractional volume of intratumoral sarcomatoid differentiation was quantified by microscopic examination of multiple histologic sections of nephrectomy specimens and the percent sarcomatoid involvement of the entire tumor was estimated to the nearest 5\% (HIST\%SARC).

\section{Magnetic resonance (MR) and data acquisition}

All MR examinations were performed on 1.5 T MRI scanners (General Electric Medical Systems, Waukesha, WI, USA or Siemens Medical Solutions, Ehrlangen, Germany) using standard pulse sequences. Dynamic T1W pre- and post-contrast images were acquired by gradient echo with fat saturation $\{$ Echo time $(\mathrm{TE})=1.5-3.3 \mathrm{~ms}$, repetition time $(\mathrm{TR})=3.3-6.7 \mathrm{~ms}$, flip angle $=10-15 \mathrm{~ms}$, slice thickness $=3.3-5.0 \mathrm{~mm}$, field of view $(\mathrm{FOV})=[299$ $498] \times[224-459] \mathrm{mm}^{2}$, matrix $=512 \times[384-512]$ or $256 \times$ [192-208], number of excitations/averages $(\mathrm{NEX})=1$, in-plane pixel resolution $0.72-1.95 \mathrm{~mm}$ \}. Post contrast imaging in the arterial phase was obtained approximately 20 seconds following contrast injection initiation of gadobutrol $0.1 \mathrm{mmol} / \mathrm{kg}$ (Bayer HealthCare, Whippany, NJ, USA). $\mathrm{T} 2 \mathrm{~W}$ non-fat saturated images were acquired by fast spinecho [effective TE $=60-184 \mathrm{~ms}$, TR $=573-1,740 \mathrm{~ms}$, slice thickness $=5-10 \mathrm{~mm}, \mathrm{FOV}=[299-489] \times[224-440] \mathrm{mm}^{2}$, matrix $=512 \times 360$ or $256 \times$ [208-256], NEX (averages) $=1$, in-plane pixel resolution $0.63-1.95 \mathrm{~mm}$ ]. Single-shot echoplanar diffusion weighted (DW) images were acquired with TE $=73-94 \mathrm{~ms}$, TR $=2,175-11,000 \mathrm{~ms}$, slice thickness $=5-$ $8 \mathrm{~mm}, \mathrm{FOV}=[319-460] \times[289-430] \mathrm{mm}^{2}$, matrix $=192 \times$ [150-156] or $256 \times 256$, NEX (averages) $=3-6$, and B-values $=50,500$, and $800 \mathrm{~s} / \mathrm{mm}^{2}$ or 50 and $800 \mathrm{~s} / \mathrm{mm}^{2}$.

Multiparameter MRI analysis was performed using software that was written in-house using MATLAB (The Mathworks, Natick, MA, USA). In the first pre-processing step, images from all sequences were interpolated to a common finest resolution and smallest slice thickness for uniformity. Next, a subtracted series (T1W arterial phase minus T1W precontrast) was spatially registered to $\mathrm{T} 2 \mathrm{~W}$ images using a novel MATLAB program to spatially co-register axial DICOM sequences. The registration algorithm used a $3 \mathrm{D}$ similarity transformation matrix rigid registration technique, similar to the coregistration technique used by previous authors (19).
The coregistration involved scaling, translation, and rotation of the $\mathrm{T} 1 \mathrm{~W}$ sequence with respect to the fixed $\mathrm{T} 2 \mathrm{~W}$ sequence. MRI sequence coregistration was evaluated for each scan based on the precision of overlap of tumor margins on axial, sagittal, and coronal reconstructions. A Likert score of 1 (unacceptable), 2 (poor), 3 (adequate), 4 (good), and 5 (near perfect) was assigned for each of the $34 \mathrm{MRI}$ scans to evaluate overall tumor margin alignment after coregistration.

After coregistration, the whole tumor and contralateral renal cortex were manually segmented on all slices of the $\mathrm{T} 2 \mathrm{~W}$ images (Figure $1 A$ ) by a dedicated oncologic radiologist. Based on thresholds derived from previous research, voxels within a patient's tumor were clustered into a "T2low" cluster if their T2W quantitative intensity was $1 \mathrm{SD}$ or more below the mean intensity of the same patient's contralateral renal cortex $(14,15)$ (Figure 1B) Voxels were also classified as "T1m" if there was mild enhancement (20-50\% increased signal) on arterial phase (Figure 1C), based on contralateral $\mathrm{T} 1 \mathrm{~W}$ precontrast cortex signal (Figure 1D), with the help of subtraction imaging (Figure $1 E)(14,15)$. In this study, we assigned mild enhancement as an increase in $\mathrm{T} 1$ pre-contrast signal by $20-50 \%$ on arterial phase based on the range of enhancement patterns observed in a series of sRCC 11 patients (14). Additionally, the arterial phase showed the most pronounced difference in degree of hypoenhancement of sRCC compared to ccRCC (14). Each sRCC tumor was sub-divided into sarcomatoid and non-sarcomatoid distinct habitats based on quantitative $\mathrm{T} 2$ and T1 arterial phase relative signals $(14,20)$. Based on the work from Takeuchi et al., we hypothesized that the T2low-T1m habitat corresponded to the sarcomatoid component of the tumor (14). The number of voxels with T2low-T1m signal divided by the total number of voxels within a segmented tumor yielded MRI\%SARC (Figure $1 F$, visualized in green). Signal intensity ratios to contralateral normal renal cortex within each patient were utilized to reduce inter-scan quantitative variability.

Areas of increased out-of-phase signal compared to inphase signal were suggestive of hemorrhage or hemosiderin and were excluded from the volume. In addition, whole tumor $\mathrm{ADC}$ values were compared between the sRCC and ccRCC cohorts but not included in the MATLAB routine due to low availability of this sequence within the sRCC cohort.

\section{Data analysis and statistical methods}

The primary endpoint of our study was to compare the MRI\%SARC value calculated from multiparameter 

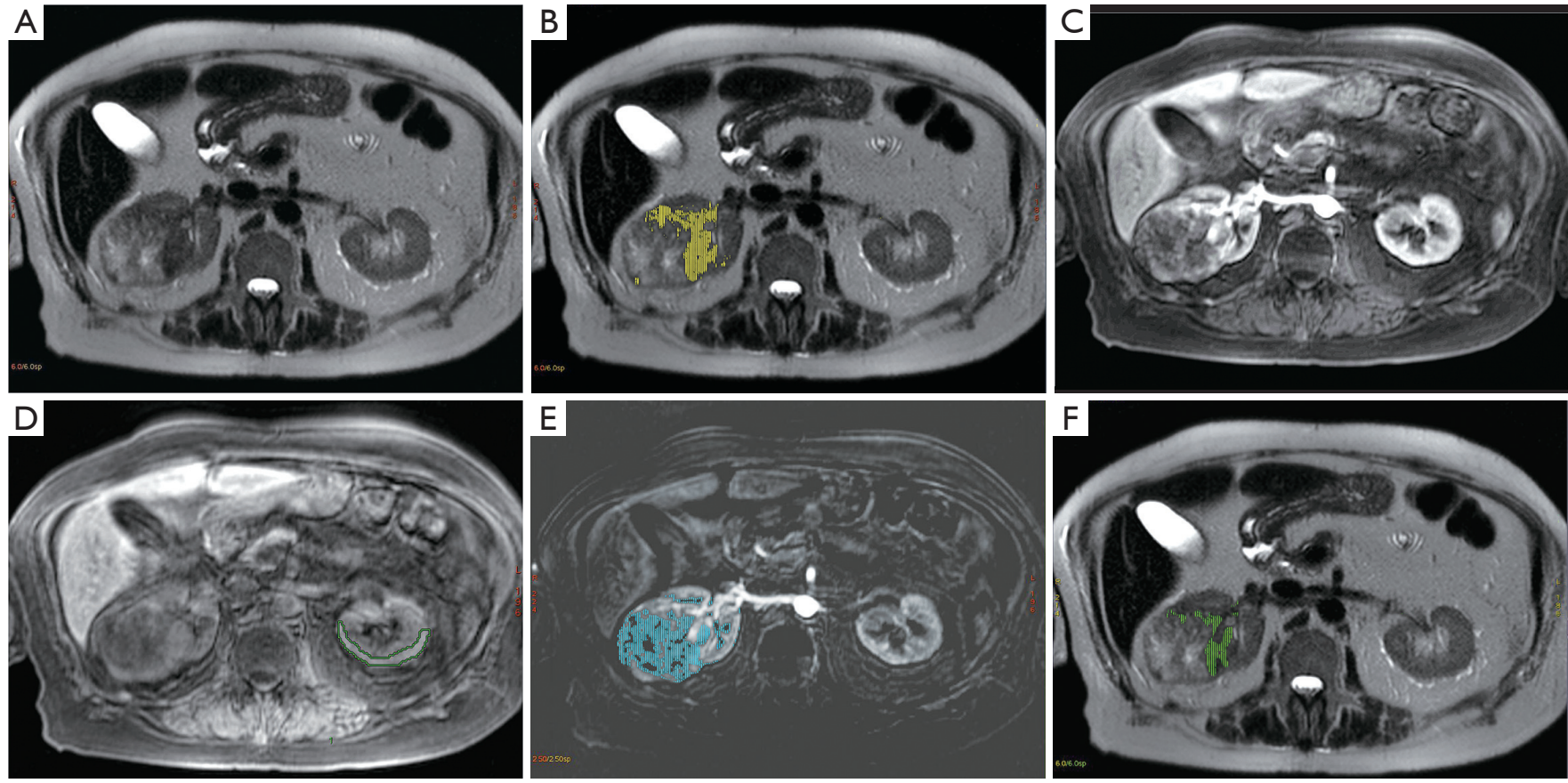

Figure $1 \mathrm{MRI}$ images showing intersection of T2W and T1W post contrast imaging meeting predefined quantitative sarcomatoid criteria. (A) Axial T2W image through the right renal mass. The low T2 signal region along the medial mass corresponds with the sarcomatoid component on this sequence; (B) axial T2W image with yellow highlighted portions representing the threshold T2 signal that is greater than one standard deviation below the contralateral (left) renal cortex T2 signal. T2low habitat is highlighted in yellow; (C) axial T1W arterial phase image shows varying degrees of enhancement within the tumor; (D) axial T1W precontrast image with contralateral normal cortex ROI segmented with a green line. Average ROI intensity =54; (E) corresponding axial T1 subtraction image (arterial phase C minus precontrast D). The threshold blue portions represent 20-50\% enhancement on the subtraction image calculated from the precontrast cortex ROI value or subtracted signal intensity of 10.8-27; (F) axial T2W image showing the intersection of T2 and T1 post contrast subtracted threshold regions or the T2low-T1m sarcomatoid habitat in green. MRI\%SARC for this whole lesion was $7 \%$ while HIST\%SARC was 20\%. ROI, region of interest; MRI\%SARC, volume fraction of sarcomatoid involvement within a tumor defined by MRI; T2W, T2-weighted; HIST\%SARC, volume fraction of sarcomatoid involvement within a tumor defined by histology; MRI\%SARC, volume fraction of sarcomatoid involvement within a tumor defined by MRI.

MRI analysis to percent sarcomatoid involvement estimated by histological examination (HIST\%SARC). Patient demographic characteristics were compared using the Mann-Whitney $U$ test or Chi-square test. Pearson correlation was used to compare MRI\%SARC and HIST\%SARC. Bland Altman and Passing-Bablok regression analysis were also used to compare the MRI quantitative model and standard histological examination. A priori limits of agreement were defined as a difference not exceeding 10 percent between the two measurements for clinical significance. The T2low-T1m habitat was also computed in control ccRCC tumors. Wilcoxon rank sum was used to compare whole lesion ADC for sRCC and control group ccRCC tumors. Statistical analysis was performed with SPSS version 24 software package (IBM Corporation, Chicago, IL, USA) and MedCalc statistical software (MedCalc Software, Ostend, Belgium).

\section{Results}

\section{Clinical characteristics}

Patient characteristics and demographics for the 34 patients included in this study are summarized in Table 1. The mean age ( \pm standard deviation) of the 17 patients diagnosed with RCC with sarcomatoid components was $60.3 \pm 11.1$ years, and 10/17 (59\%) were male. The mean age of the 17 control patients with ccRCC was $64.5 \pm 7.6$ years, and $10 / 17$ (59\%) were male. The median tumor size by greatest dimension in 
Table 1 Cohort demographics and radiologic/pathologic features

\begin{tabular}{|c|c|c|c|}
\hline Tumor features & $\begin{array}{l}\text { sRCC } \\
(n=17)\end{array}$ & $\begin{array}{c}\text { ccRCC } \\
(n=17)\end{array}$ & $P$ \\
\hline Mean age (years) & 60.3 & 64.5 & 0.413 \\
\hline Sex (males) & 10 & 10 & 1 \\
\hline Median tumor size by pathology $(\mathrm{cm})$ & 11 & 9 & 0.493 \\
\hline Median tumor size by MRI (cm) & 9.8 & 9 & 0.731 \\
\hline Pathologic tumor category & & & 0.21 \\
\hline T1 and T2 & 3 & 7 & \\
\hline T3 & 13 & 8 & \\
\hline $\mathrm{T} 4$ & 1 & 2 & \\
\hline Pathologic $\mathrm{N}$ category & & & $0.023^{*}$ \\
\hline No & 7 & 5 & \\
\hline N1 & 5 & 1 & \\
\hline N2 & 2 & 0 & \\
\hline NX & 3 & 11 & \\
\hline Clinical metastasis & & & 1 \\
\hline Negative (M0) or unknown & 9 & 9 & \\
\hline Positive (M1) & 8 & 8 & \\
\hline Perineural invasion & 2 & 0 & 0.145 \\
\hline Lympho-vascular invasion & 10 & 6 & 0.169 \\
\hline Renal capsule invasion & 13 & 8 & 0.078 \\
\hline Tumor necrosis & & & $0.008^{*}$ \\
\hline $0 \%$ & 0 & 8 & \\
\hline $1-20 \%$ & 10 & 4 & \\
\hline $21-40 \%$ & 5 & 4 & \\
\hline $41-60 \%$ & 2 & 0 & \\
\hline$>60 \%$ & 0 & 1 & \\
\hline
\end{tabular}

*, significant difference. RCC, renal cell carcinoma; MRI, magnetic resonance imaging; ccRCC, clear cell renal cell carcinoma.

the sRCC group by gross pathologic examination was $11 \mathrm{~cm}$ [interquartile range (IQR): $8.5-14 \mathrm{~cm}$ )], while the median tumor size by gross examination in the ccRCC group was $9 \mathrm{~cm}$ (IQR: 4-11 cm). By MRI measurements, median tumor size in sRCC patients was smaller than gross pathology measurements at $9.8 \mathrm{~cm}$ (IQR: $8.2-11.5 \mathrm{~cm}$ ). Median tumor size in ccRCC patients by MRI was the same as measured by gross examination (IQR: $4.7-10.7 \mathrm{~cm}$ ). However, there was no significant difference in tumor size by measuring by gross exam or MRI in the sRCC group $(\mathrm{P}=0.493)$ and there were no significant differences in tumor size between sRCC and ccRCC groups $(\mathrm{P}=0.493$ and 0.731 by pathology and MRI, respectively).

At the time of surgical resection, 8/17 (47\%) patients in each of the sRCC and ccRCC groups had known or presumed clinical metastatic disease. Within the sRCC group, 8/17 (47\%) had underlying ccRCC pathology and $3 / 17$ (18\%) had underlying chromophobe RCC. Five of 17 patients $(29 \%)$ had tumors with predominant sarcomatoid changes without recognizable epithelial component and were categorized as unclassified RCC with sarcomatoid change, and one patient had poorly differentiated underlying pathology. Two patients within the sRCC group (12\%) had documented perineural invasion, but there was no perineural invasion noted in the ccRCC group. While 10/17 (59\%) patients with sRCC was noted to have lympho-vascular invasion, only $6 / 17$ (35\%) patients with ccRCC had lympho-vascular invasion. Furthermore, $13 / 17(76 \%)$ of sRCC patients were found to have renal capsule invasion, while 8/17 (47\%) of ccRCC patients had capsule invasion. Some degree of tumor necrosis was found in all 17 sRCC patients, while 9/17 (53\%) of ccRCC patients were noted to have tumor necrosis. Of these clinical characteristics, only tumor necrosis was found to be significantly different between the sRCC and ccRCC groups $(\mathrm{P}=0.008)$.

\section{Quantitative MRI characteristics}

Table 2 displays quantitative MRI characteristics between the sRCC and ccRCC groups. There was no significant difference in the average relative $\mathrm{T} 1 \mathrm{~W}$ arterial phase enhancement between the groups $(\mathrm{P}=0.18)$. Mean $\mathrm{T} 2 \mathrm{~W}$ intensity values did not significantly differ between the two groups $(\mathrm{P}=0.39)$, although the percentage of T2low volume in the sRCC group was significantly greater than in the ccRCC group $(\mathrm{P}=0.025)$. Whole lesion mean ADC values also differed significantly between the two groups, with the mean ADC of the sRCC group of $(1.265 \pm 1.88) \times 10^{-3} \mathrm{~mm}^{2} / \mathrm{s}$ and the mean ADC of the ccRCC group $(1.563 \pm 1.95) \times 10^{-3} \mathrm{~mm}^{2} / \mathrm{s}(\mathrm{P}=0.005)$. Average percent sarcomatoid MRI values generated by multiparameter MRI habitat analysis produced a significant difference between sRCC and ccRCC groups $(\mathrm{P}<0.001)$.

Figure 2 demonstrates different statistical methods used to compare percent sarcomatoid values obtained by pathologic examination and MRI in the sRCC group. 
Table 2 Quantitative MRI lesion analysis

\begin{tabular}{|c|c|c|c|}
\hline MRI features & sRCC & $\mathrm{ccRCC}$ & $P$ \\
\hline Mean signal T2 mass/T2 contra cortex & 0.86 & 1.11 & $0.024^{*}$ \\
\hline$\%$ mass with $\mathrm{T} 2$ signal $<$ contra renal cortex & 0.62 & 0.39 & $0.025^{\star}$ \\
\hline Mean ADC values $\left(\times 10^{-3} \mathrm{~mm}^{2} / \mathrm{s}\right)$ & $1.265 \pm 1.88$ & $1.563 \pm 1.95$ & $0.005^{*}$ \\
\hline
\end{tabular}

*, significant difference. RCC, renal cell carcinoma; ADC, apparent diffusion coefficient; MRI\%SARC, volume fraction of sarcomatoid involvement within a tumor defined by MRI; MRI, magnetic resonance imaging; ccRCC, clear cell renal cell carcinoma; sRCC, sarcomatoid differentiation in renal cell carcinoma.
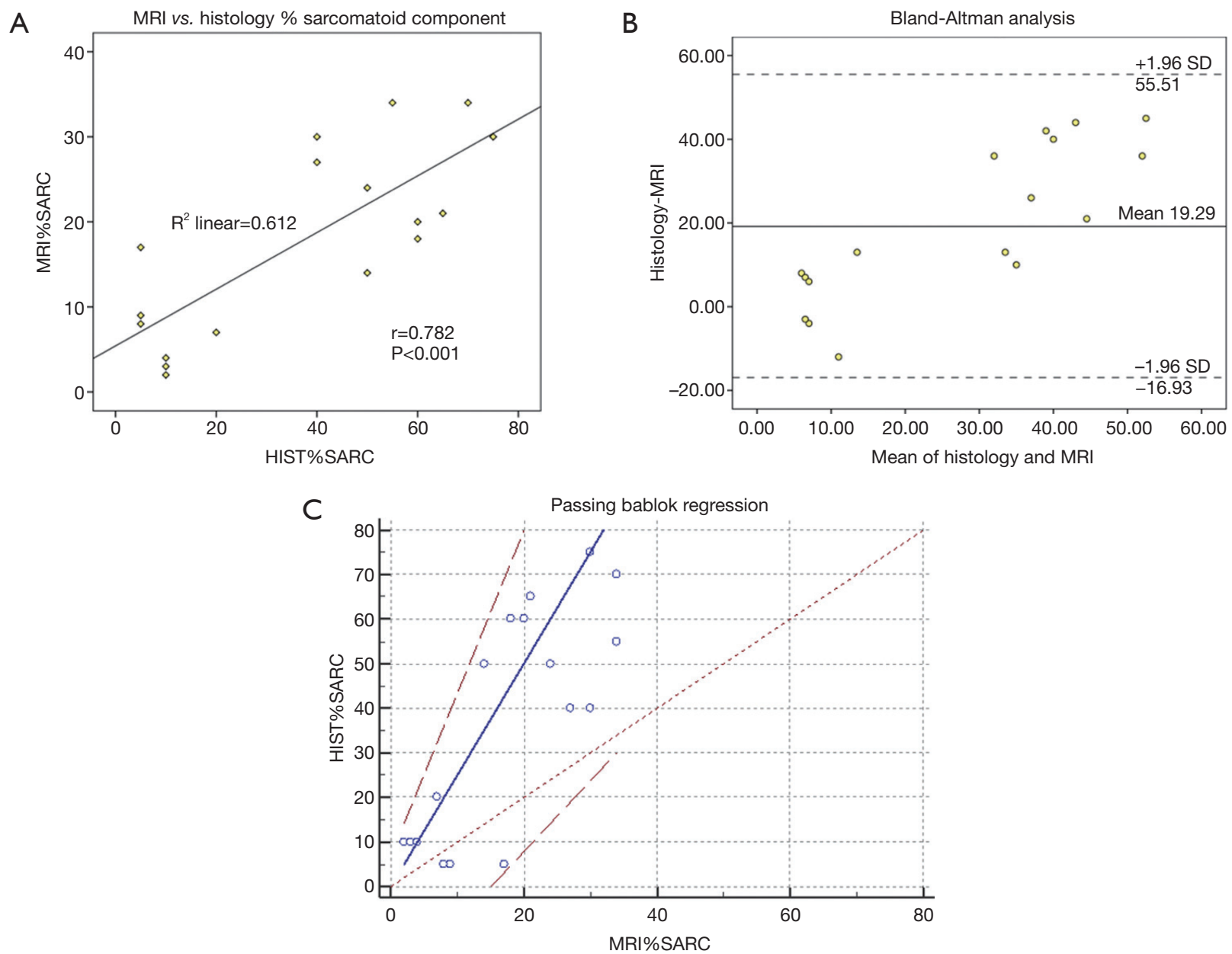

Figure 2 Statistical analyses of MRI\%SARC compared to HIST\%SARC. (A) Scatter plot of percent sarcomatoid involvement calculated by quantitative MRI analysis versus standard estimation by histology shows strong correlation coefficient, $r=0.782$ and $\mathrm{R}^{2}=0.612, \mathrm{P}<0.001 ;(\mathrm{B})$ bland Altman analysis shows proportional bias and limits of agreement (bias 19.29, limits of agreement -16.93 to 55.51). Results suggest MRI\%SARC underestimates Histology\%SARC by $19 \%$ on average; (C) Passing-Bablok regression analysis shows y=0+2.5x. CI lines are dashed (long). A linear relationship between the two methods is noted, with the intercept of 0 (95\% CI: -24.09 to 6.82). The slope is 2.5 (95\% CI: 1.59-3.64), which excludes 1 and suggests a proportional difference between methods. MRI, magnetic resonance imaging; HIST\%SARC, volume fraction of sarcomatoid involvement within a tumor defined by histology; MRI\%SARC, volume fraction of sarcomatoid involvement within a tumor defined by MRI. 

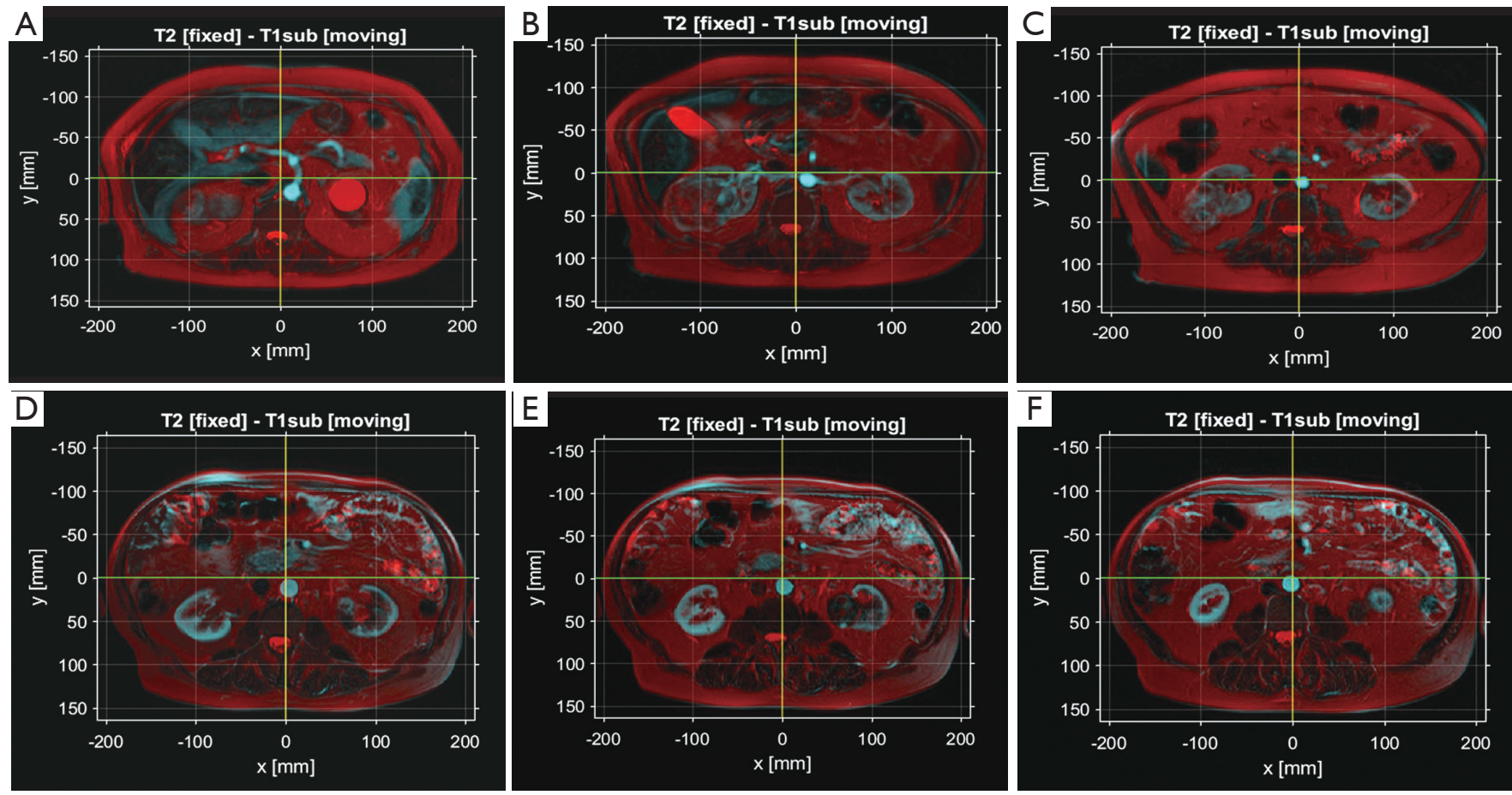

Figure 3 Coregistration of MRI sequences. (A,B,C) Axial coregistered images from superior to inferior through an sRCC lesion. Red color represents the fixed T2 image while superimposed light blue represents the transformed T1 (arterial minus pre) subtraction signal. Coregistration Likert score 5 was assigned for this case for near perfect co-registration; (D,E,F) axial coregistered images from superior to inferior through a different ccRCC lesion. Coregistration Likert score 4 was assigned for this case for good co-registration. ccRCC, clear cell renal cell carcinoma.

Pearson correlation analysis revealed a strong association between the calculated percent sarcomatoid MRI value (MRI\%SARC) derived from quantitative MRI analysis and estimated percent sarcomatoid involvement determined by histological examination (HIST\%SARC) (Figure 2A, $\mathrm{r}=0.782, \mathrm{P}<0.001)$. Figure $2 B$ demonstrates the distribution plot of differences between percent sarcomatoid measurements by MRI and histologic examination. BlandAltman analysis demonstrated proportional bias, with a mean bias of 19.29 (95\% CI: 9.79-28.79) and with $95 \%$ limits of agreement of -16.93 (95\% CI: -33.38 to -0.48 ) to 55.51 (95\% CI: 39.06-71.96), suggesting MRI\% SARC underestimated values compared to HIST\%SARC by $19 \%$. To quantify the proportional difference, Passing Bablock regression was performed which suggested a linear relationship between the two methods, with the intercept of 0 (95\% CI: -24.09 to 6.82$)$ and slope of 2.5 (95\% CI: 1.59-3.64) (Figure 2C). CUSUM test for linearity indicated no significant deviation from linearity $(\mathrm{P}=0.96)$.

MRI coregistration evaluation of the 34 MRI scans yielded Likert scores of: 5 near perfect ( $\mathrm{n}=17)$; 4 good $(\mathrm{n}=14) ; 3$ acceptable $(\mathrm{n}=1) ; 2$ poor or 1 unacceptable $(\mathrm{n}=0)$. Figure 3 shows examples of MRI coregistration classified as Likert score 5 (Figure $3 A, B, C$ ) and Likert score 4 (Figure $3 D, E, F$ ) cases in this study. A single MRI case was graded as three but still acceptable for analysis.

\section{Discussion}

We calculated the percent volume of sarcomatoid involvement in RCC tumors on MRI using relative thresholds based on previous research (MRI\%SARC). Compared to histology, MRI\%SARC showed strong correlation with histology but poor agreement. MRI\%SARC significantly underestimated percent sarcomatoid values generated by histologic exam, and our MATLAB routine did not achieve acceptable limits of agreement. Comparison of MRI quantification and histology revealed a proportional difference between the two methods. Strong correlation of the T2low-T1m habitat volume with 
the histological sarcomatoid component shows promise that this habitat volume corresponds to the sarcomatoid volume. To our knowledge, our study was the first endeavor to quantify sarcomatoid transformation by volume fraction with MRI in RCC. Given the poor prognosis of sRCCs, early identification of sarcomatoid transformation would confer a significant advantage in coordinating clinical management. RCC tumors containing $\geq 30 \%$ sarcomatoid differentiation were $52 \%$ more likely to die from RCC compared with patients whose tumors contained $<30 \%$ sarcomatoid differentiation (9). Additionally, tissue sampling in RCC is hampered by tissue heterogeneity within solid tumors and this can lead to false negative biopsies and potentially poorer prognoses $(4,13,21)$. In a study of 166 patients who underwent percutaneous biopsy prior to cytoreductive nephrectomy for metastatic RCC, Abel et al. found only 4 of 34 nephrectomy specimens with sarcomatoid features were identified pre-operatively (22). Thus, pre-operative imaging presents a promising opportunity to non-invasively elucidate the underlying histopathology that constitutes sarcomatoid RCC.

Several caveats require attention prior to further discussion regarding the results of our study. The major limitations of this study were the retrospective investigation and small sample size, which was restricted by the low incidence of sRCC patients who also received pre-operative MRI. Our results are an encouraging validation that multiparametric MRI can identify sarcomatoid components in RCC prior to surgical resection with quantification that correlates with histology, but the small sample precluded desirable study design features. For instance, our study utilized previously reported MRI parameter thresholds which compared sRCC patients to ccRCC patients and so this study also limited the control group to consecutively chosen ccRCC patients (14). While ccRCC is the predominant subtype among RCCs, papillary and chromophobe subtypes often demonstrate T2 low signal areas. Additionally, quantitative values derived from MRI can be affected by the parameters and techniques used. In this study, we assigned mild enhancement as increase in T1 pre-contrast signal by $20-50 \%$ on arterial phase based on the range of enhancement patterns observed in a series of 11 sRCC patients (14). Given the limited availability of large sRCC cohorts with dynamic post contrast data, Takeuchi et al. study served as a suitable cohort from which to derive quantitative enhancement values (14). Future larger prospective studies should include subsets of other RCC subtypes and may be able to derive and validate optimal threshold values dependent on imaging protocols.

Accordingly, several technical matters are notable in our study. The sRCC group mean ADC value of $1.265 \times 10^{-3} \mathrm{~mm}^{2} / \mathrm{s}$ in our study was greater than the mean ADC value found in the study from Takeuchi $e t$ al. of $0.860 \times 10^{-3} \mathrm{~mm}^{2} / \mathrm{s}$, but lower than their reported background or clear cell ADC of $1.950 \times 10^{-3} \mathrm{~mm}^{2} / \mathrm{s}$ (14). Takeuchi et al. utilized manual regions of interest (ROI) targeting sarcomatoid or background regions based on T2 signal, whereas our study used whole lesion ROIs (14). Our sRCC group ADC values represent whole lesion techniques capturing a mixture of both sarcomatoid and background or clear cell components and correspondingly, our ADC values fell between previously reported sRCC and ccRCC values.

Co-registration of multiple MRI sequences is another challenge for quantitative multiparametric analysis. Patient motion or inconsistent breath holding technique could lead to spatial differences in sequences which would negatively affect our volume calculation. Furthermore, receiver coil related field inhomogeneities within the $\mathrm{T} 2$ and $\mathrm{T} 1$ post contrast sequences could lead to regional differences in quantification of MR signal. Future studies utilizing MR fingerprinting which includes quantitative $\mathrm{T} 1, \mathrm{~T} 2$, and B1 maps obtained within a single breath hold may provide a solution (23). Nevertheless, our MATLAB routine addressed spatial co-registration and intra-scan ratios were utilized to reduce potential errors. Qualitative radiologist assessment helped to confirm there were no clinically significant areas of in-homogeneity within tumor regions.

Finally, another explanation for the differences between MRI\%SARC and HIST\%SARC could be related to sampling error due to the selective nature of the histological examination, with the possibility that HIST\%SARC overestimated the true sarcomatoid component. In the histological exam, 5-8 sections from each tumor were selected to be representative of the entire tumor. Unlike MRI exams, it is not clinically feasible or practical to analyze entire tumor specimens microscopically. Our study methods demonstrate how pre-operative advanced imaging techniques may be able to elucidate underlying tumor habitats which can help direct tissue analysis. Future prospective radiological-pathological studies could spatially register thin-slice gross tumor sections and their focal microscopic correlates with MRI exams to provide a histopathological map of the resected gross specimen to provide more precision for both quantitative MRI thresholds and histopathologic diagnosis. Furthermore, studies with 
larger sample sizes may utilize multivariate analysis of clinical characteristics including tumor size and peritumoral neovascularity in addition to quantitative MRI criteria to improve the agreement between MRI and histology.

\section{Conclusions}

Multiparametric MRI analysis to quantify sarcomatoid features in RCC correlates with standard histological examination but underestimates sarcomatoid involvement. Future research with larger sRCC cohorts may provide optimized relative MRI signal thresholds corresponding to the sarcomatoid component which could aide in the preoperative diagnosis of clinically significant high percentage sarcomatoid involvement RCC.

\section{Acknowledgements}

None.

\section{Footnote}

Conflicts of Interest: The authors have no conflicts of interest to declare.

Ethical Statement: The study was approved by the Institutional Review Board (Chesapeake/Advarra IRB protocol 00014728) and waiver of consent was granted.

\section{References}

1. Choueiri TK, Motzer RJ. Systemic Therapy for Metastatic Renal-Cell Carcinoma. N Engl J Med 2017;376:354-66.

2. Bedke J, Gauler T, Grunwald V, Hegele A, Herrmann E, Hinz S, Janssen J, Schmitz S, Schostak M, Tesch H, Zastrow S, Miller K. Systemic therapy in metastatic renal cell carcinoma. World J Urol 2017;35:179-88.

3. Low G, Huang G, Fu W, Moloo Z, Girgis S. Review of renal cell carcinoma and its common subtypes in radiology. World J Radiol 2016;8:484-500.

4. Shuch B, Bratslavsky G, Linehan WM, Srinivasan R. Sarcomatoid renal cell carcinoma: a comprehensive review of the biology and current treatment strategies. Oncologist 2012;17:46-54.

5. Cheville JC, Lohse CM, Zincke H, Weaver AL, Leibovich BC, Frank I, Blute ML. Sarcomatoid renal cell carcinoma: an examination of underlying histologic subtype and an analysis of associations with patient outcome. Am J Surg
Pathol 2004;28:435-41.

6. Kunene V, Miscoria M, Pirrie S, Islam MR, Afshar M, Porfiri E. Sarcomatoid renal cell carcinoma: clinical outcome and survival after treatment with sunitinib. Clin Genitourin Cancer 2014;12:251-5.

7. Dutcher JP. Recent developments in the treatment of renal cell carcinoma. Ther Adv Urol 2013;5:338-53.

8. Kyriakopoulos CE, Chittoria N, Choueiri TK, Kroeger N, Lee JL, Srinivas S, Knox JJ, Bjarnason GA, Ernst SD, Wood LA, Vaishampayan UN, Agarwal N, Pal SK, Kanesvaran R, Rha SY, Yuasa T, Donskov F, North SA, Heng DY, Rini BI. Outcome of patients with metastatic sarcomatoid renal cell carcinoma: results from the International Metastatic Renal Cell Carcinoma Database Consortium. Clin Genitourin Cancer 2015;13:e79-85.

9. Zhang BY, Thompson RH, Lohse CM, Leibovich BC, Boorjian SA, Cheville JC, Costello BA. A novel prognostic model for patients with sarcomatoid renal cell carcinoma. BJU Int 2015;115:405-11.

10. Adibi M, Thomas AZ, Borregales LD, Merrill MM, Slack RS, Chen HC, Sircar K, Murugan P, Tamboli P, Jonasch E, Tannir NM, Matin SF, Wood CG, Karam JA. Percentage of sarcomatoid component as a prognostic indicator for survival in renal cell carcinoma with sarcomatoid dedifferentiation. Urol Oncol 2015;33:427.e17-23.

11. de Peralta-Venturina M, Moch H, Amin M, Tamboli P, Hailemariam S, Mihatsch M, Javidan J, Stricker H, Ro JY, Amin MB. Sarcomatoid differentiation in renal cell carcinoma: a study of 101 cases. Am J Surg Pathol 2001;25:275-84.

12. Shuch B, Said J, La Rochelle JC, Zhou Y, Li G, Klatte T, Kabbinaavar FF, Pantuck AJ, Belldegrun AS. Cytoreductive nephrectomy for kidney cancer with sarcomatoid histology--is up-front resection indicated and, if not, is it avoidable? J Urol 2009;182:2164-71.

13. Schieda N, Thornhill RE, Al-Subhi M, McInnes MD, Shabana WM, van der Pol CB, Flood TA. Diagnosis of Sarcomatoid Renal Cell Carcinoma With CT: Evaluation by Qualitative Imaging Features and Texture Analysis. AJR Am J Roentgenol 2015;204:1013-23.

14. Takeuchi $M$, Urano $M$, Hara $M$, Fujiyoshi $Y$, Inagaki $H$, Shibamoto Y. Characteristic MRI findings of sarcomatoid renal cell carcinoma dedifferentiated from clear cell renal carcinoma: radiological-pathological correlation. Clin Imaging 2013;37:908-12.

15. Takeuchi M, Kawai T, Suzuki T, Naiki T, Kawai N, Fujiyoshi Y, Inagaki H, Kohri K, Hara M, Shibamoto Y. MRI for differentiation of renal cell carcinoma with 
sarcomatoid component from other renal tumor types. Abdom Imaging 2015;40:112-9.

16. Mickisch GH, Garin A, van Poppel H, de Prijck L, Sylvester R. Radical nephrectomy plus interferon-alfabased immunotherapy compared with interferon alfa alone in metastatic renal-cell carcinoma: a randomised trial. Lancet 2001;358:966-70.

17. Blute ML, Leibovich BC, Cheville JC, Lohse CM, Zincke H. A protocol for performing extended lymph node dissection using primary tumor pathological features for patients treated with radical nephrectomy for clear cell renal cell carcinoma. J Urol 2004;172:465-9.

18. Kutikov A, Uzzo RG, Caraway A, Reese CT, Egleston BL, Chen DY, Viterbo R, Greenberg RE, Wong YN, Raman JD, Boorjian SA. Use of systemic therapy and factors affecting survival for patients undergoing cytoreductive nephrectomy. BJU Int 2010;106:218-23.

Cite this article as: Jeong D, Raghunand N, Hernando D, Poch M, Jeong K, Eck B, Dhillon J. Quantification of sarcomatoid differentiation in renal cell carcinoma on magnetic resonance imaging. Quant Imaging Med Surg 2018;8(4):373382. doi: 10.21037/qims.2018.04.09
19. Will S, Martirosian P, Wurslin C, Schick F. Automated segmentation and volumetric analysis of renal cortex, medulla, and pelvis based on non-contrast-enhanced T1and T2-weighted MR images. Magma 2014;27:445-54.

20. Gatenby RA, Grove O, Gillies RJ. Quantitative imaging in cancer evolution and ecology. Radiology 2013;269:8-15.

21. Marusyk A, Polyak K. Tumor heterogeneity: causes and consequences. Biochim Biophys Acta 2010;1805:105-17.

22. Abel EJ, Culp SH, Matin SF, Tamboli P, Wallace MJ, Jonasch E, Tannir NM, Wood CG. Percutaneous biopsy of primary tumor in metastatic renal cell carcinoma to predict high risk pathological features: comparison with nephrectomy assessment. J Urol 2010;184:1877-81.

23. Chen Y, Jiang Y, Pahwa S, Ma D, Lu L, Twieg MD, Wright KL, Seiberlich N, Griswold MA, Gulani V. MR Fingerprinting for Rapid Quantitative Abdominal Imaging. Radiology 2016;279:278-86. 